\title{
THE PROFILE MINIMIZATION PROBLEM IN TREES*
}

\author{
DAVID KUO ${ }^{\dagger}$ AND GERARD J. CHANG ${ }^{\dagger}$
}

\begin{abstract}
The profile minimization problem is to find a one-to-one function $f$ from the vertex set $V(G)$ of a graph $G$ to the set of all positive integers such that $\sum_{x \in V(G)}\left\{f(x)-\min _{y \in N[x]} f(y)\right\}$ is as small as possible, where $N[x]=\{x\} \cup\{y: y$ is adjacent to $x\}$ is the closed neighborhood of $x$ in $G$. This paper gives an $O\left(n^{1.722}\right)$ time algorithm for the problem in a tree of $n$ vertices.
\end{abstract}

Key words. sparse matrix, profile, labeling, tree, leaf, centroid, basic path, algorithm

AMS subject classifications. 05C78, 05C85, 68R10

1. Introduction. The profile minimization problem was introduced by [5], [6] as a technique for handling sparse matrices. For instance, in the finite element method [8], [9], we want to solve a system of linear equations $A x=b$ where $A$ is a sparse symmetric $n \times n$ matrix. Suppose for each row $i, a_{i i} \neq 0$ and $p_{i}$ is the position of the first non-zero element in this row. We call

$$
w_{i}=i-p_{i}=i-\min \left\{j: a_{i j} \neq 0\right\}
$$

the width of row $i$, and call

$$
P(A)=\sum_{i=1}^{n} w_{i}
$$

the profile of matrix $A$. To store $A$, we need only store $w_{i}+1$ elements in each row $i$, which are from position $p_{i}$ to position $i$. The total amount of storage for this scheme is then $P(A)+n$. In order to reduce the amount of storage, we need only permute the rows and columns of $A$ simultaneously such that the resulting matrix has minimum profile, i.e., we need to find a permutation matrix $Q$ such that the profile $P\left(Q A Q^{t}\right)$ is minimized.

We can reformulate this problem in terms of graphs. Associate the matrix $A$ with a graph $G$ such that $V(G)=\left\{v_{1}, v_{2}, \ldots, v_{n}\right\}$ and $E(G)=\left\{\left(v_{i}, v_{j}\right): i \neq j\right.$ and $\left.a_{i j} \neq 0\right\}$. Note that

$$
P(A)=\sum_{i=1}^{n} w_{i}=\sum_{i=1}^{n}\left(i-\min _{v_{j} \in N\left[v_{i}\right]} j\right),
$$

where $N\left[v_{i}\right] \equiv\left\{v_{i}\right\} \cup\left\{v_{j}: v_{i}\right.$ is adjacent to $\left.v_{j}\right\}$ is the closed neighborhood of $v_{i}$ in $G$. The row and column permutation $Q$ corresponds to a one-to-one function $f$ from $V(G)$ onto $\{1,2, \ldots, n\}$ and $P\left(Q A Q^{t}\right)=\sum_{x \in V(G)}\left(f(x)-\min _{y \in N[x]} f(y)\right)$. This motivates the definition of the profile of a graph given below.

For technical reasons, however, we shall give a slightly more general definition than that described in the previous paragraph. A labeling of a graph $G$ is a one-to-one function $f$ from the vertex set $V(G)$ to the set of all positive integers. A labeling is simple if it maps $V(G)$ onto $\{1,2, \ldots,|V(G)|\}$. For a labeling $f$, the profile-width of a vertex $x$ is defined as

$$
w_{f}(x)=f(x)-\min _{y \in N[x]} f(y) .
$$

${ }^{*}$ Received by the editors March 11, 1991; accepted for publication (in revised form) July 30, 1992. This work was supported in part by the National Science Council of the Republic of China under grant NSC79-0208-M009-31.

${ }^{\dagger}$ Institute of Applied Mathematics, National Chiao Tung University, Hsinchu 30050, Taiwan, Republic of China (gjchang@cc.nctu.edu.tw). 
The profile of $G$ with respect to $f$ is

$$
P_{f}(G)=\sum_{x \in V} w_{f}(x)
$$

and the profile of $G$ is

$$
P(G)=\min \left\{P_{f}(G): f \text { is a labeling of } G\right\} .
$$

A labeling $f$ is optimal if $P_{f}(G)=P(G)$.

The purpose of this paper is to study the profile minimization problem, i.e., the problem of determining the profile $P(G)$ of a graph $G$, from an algorithmic point of view. The profile minimization problem is analogous to the linear arrangement problem, which is to find a labeling $f$ of a graph $G$ such that $\sum\{|f(x)-f(y)|:(x, y)$ is an edge in $G\}$ is minimized (see [1], [3], [7]). Reference [5] proved that the profile minimization problem is equivalent to the problem of interval graph completion, which is known to be NP-complete even when $G$ is stipulated to be an edge graph (see [4]). The main result of this paper is to give an $O\left(n^{1.722}\right)$ time algorithm for the problem when $G$ is a tree of $n$ vertices.

The rest of this paper is organized as follows. In $\S 2$, we establish several basic properties that motivate the development of our algorithm. In particular, we prove that for a tree $T$ there exists a basic path $\alpha(x, y)$ such that $P(T)=P(T-\alpha(x, y))+|E(T)|$. So the problem becomes that of finding a path $\alpha(x, y)$ such that $P(T-\alpha(x, y))$ is minimized. For the purposes of recurrence, we also introduce the problem of finding a path $\alpha(x, y)$ such that $P(T-\alpha(x, y))$ is minimized, with the boundary condition that $y$ is fixed. In order to determine the basic path, $\S 3$ develops theorems that narrow the possibilities for the basic path. For instance, we prove that $\alpha(x, y)$ contains centroids of the tree. This also means that the number of vertices of each component of $T-\alpha(x, y)$ is no more than half the number of vertices of $T$. This is important in determining the speed of our recursive algorithm. Section 4 uses these results to design an algorithm, and $\S 5$ analyzes the time complexity of the algorithm.

2. Motivating properties. This section shows the existence of a basic path $\alpha(x, y)$ such that $P(T)=P(T-\alpha(x, y))+|E(T)|$ and introduces the problem of finding a minimum such path with the boundary condition that $y$ is fixed. The following properties are obvious and their proofs are omitted.

PROPOSITION 2.1. An optimal labeling of a connected graph $G$ maps $V(G)$ onto a set of consecutive integers.

PROPOSITION 2.2 ([5]). If $H$ is a subgraph of $G$, then $P(H) \leq P(G)$.

PROPOSITION 2.3 ([5]). If $G$ has $m$ components $G_{1}, G_{2}, \ldots, G_{m}$, then $P(G)=\sum_{i=1}^{m}$ $P\left(G_{i}\right)$.

We can in fact assume that an optimal labeling of a graph is simple even if it is not connected. Suppose $T$ is a tree of $n$ vertices. For any leaf $x$ and any vertex $y$ in $T$, consider the unique $(x, y)$-path $\alpha(x, y)=\left(v_{0}, v_{1}, \ldots, v_{r}\right)$, where $v_{0}=x$ and $v_{r}=y$. Suppose that for each $i, 1 \leq i \leq r, T-\alpha(x, y)$ has $n_{i}$ components $T_{i 1}, T_{i 2}, \ldots, T_{i n_{i}}$ each with a vertex $v_{i j}$ adjacent to $v_{i}$ in $T$ (see Fig. 2.1). Let $f_{i}$ be an optimal simple labeling of $F_{i}=\cup_{1 \leq j \leq n_{i}} T_{i j}$. We consider a simple labeling $f_{x y}$ defined by

$$
f_{x y}(v)= \begin{cases}1 & \text { if } v=v_{0}, \\ f_{x y}\left(v_{i-1}\right)+f_{i}(v) & \text { if } v \in V\left(F_{i}\right), \\ f_{x y}\left(v_{i-1}\right)+\left|V\left(F_{i}\right)\right|+1 & \text { if } v=v_{i} .\end{cases}
$$


See Fig. 2.2 for an example of $f_{x y}$ with $\alpha(x, y)=(a, b, c, d)$. Note that the numbers beside the vertices are their labels. Then

$$
\begin{aligned}
& w_{f_{y,}}\left(v_{0}\right)=0, \\
& w_{f_{x y}}\left(v_{i}\right)=f_{x y}\left(v_{i}\right)-f_{x y}\left(v_{i-1}\right)=\left|V\left(F_{i}\right)\right|+1 \quad \text { for } 1 \leq i \leq r,
\end{aligned}
$$

and

$$
w_{f_{y .}}(v)=w_{f_{i}}(v) \quad \text { for } v \in V\left(F_{i}\right)
$$

Consequently

$$
\begin{aligned}
P_{f_{x y}}(T) & =\sum_{i=1}^{r}\left(\left|V\left(F_{i}\right)\right|+1+P\left(F_{i}\right)\right) \\
& =|E(T)|+\sum_{i=1}^{r} P\left(F_{i}\right) \\
& =|E(T)|+\sum_{i=1}^{r} \sum_{j=1}^{n_{i}} P\left(T_{i j}\right)
\end{aligned}
$$

We call $\alpha(x, y)$ the basic path (with respect to the labeling $f_{x y}$ ). Note that

$$
1=f_{x y}\left(v_{0}\right)<f_{x y}\left(v_{1}\right)<\cdots<f_{x y}\left(v_{r}\right)=n .
$$

In general, an optimal labeling of a tree is of this type.

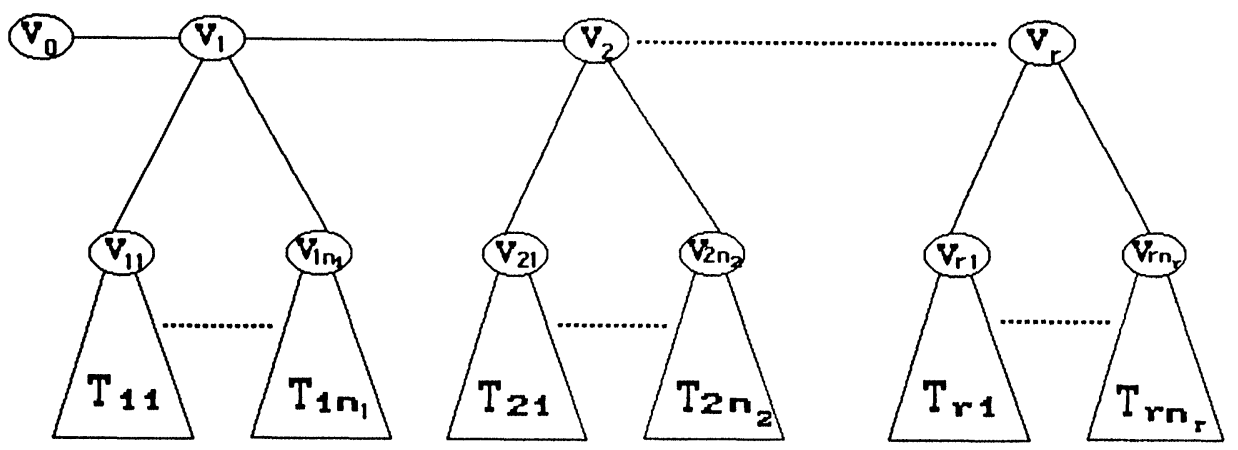

FIG. 2.1. Tree $T$.

THEOREM 2.4. If $f$ is an optimal labeling of a tree $T$ of $n$ vertices, then $f=f_{x y}$ where $x=f^{-1}(1)$ is a leaf and $y=f^{-1}(n)$ is adjacent to at most one non-leaf vertex.

Proof. Let $\alpha(z, u)=\left(v_{0}, v_{1}, \ldots, v_{r}\right)$ be a longest path containing both $x$ and $y$, say, $x=v_{s}$ and $y=v_{t}$ for $0 \leq s<t \leq r$. Note that since $r$ is the maximum, $v_{0}$ and $v_{r}$ are leaves. In this case $n_{r}=0$ and $P_{f_{z u}}(T)=P_{f_{z u^{\prime}}}(T)$, where $u^{\prime}=v_{r-1}$.

Suppose $T$ and $\alpha(z, u)$ are as shown in Fig. 2.1. Let $f_{i j}=\left.f\right|_{V\left(T_{i j}\right)}$ be the labeling $f$ restricted on $V\left(T_{i j}\right)$. Then, by definition,

$$
P(T)=P_{f}(T) \geq \sum_{i=0}^{r} w_{f}\left(v_{i}\right)+\sum_{i=1}^{r} \sum_{j=1}^{n_{i}} P_{f_{i j}}\left(T_{i j}\right) .
$$




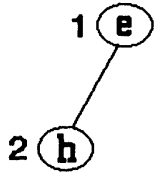

$T_{11}$

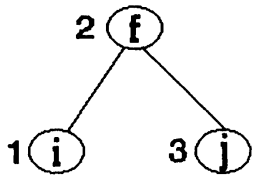

$\mathrm{T}_{12}$

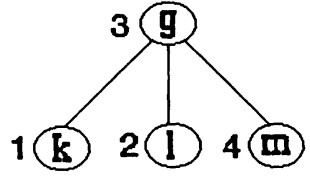

$\mathrm{T}_{31}$

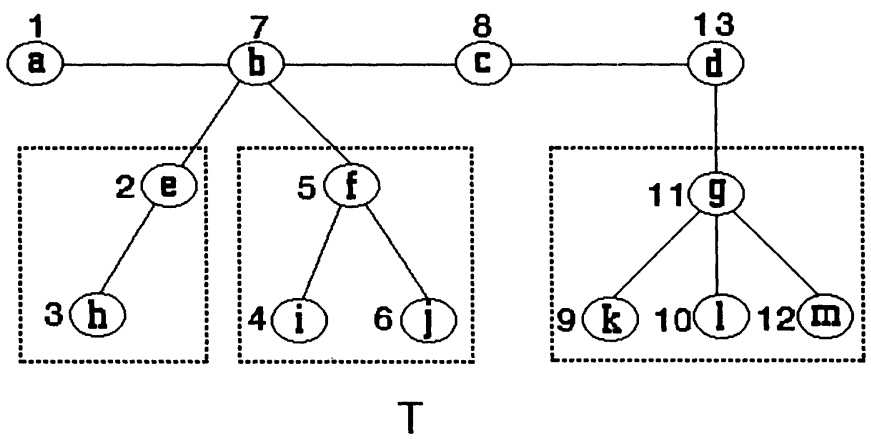

FIG. 2.2. An example of $f_{x y}$.

Note that

$$
\sum_{i=0}^{r} w_{f}\left(v_{i}\right) \geq \sum_{i=s+1}^{t} w_{f}\left(v_{i}\right) \geq \sum_{i=s+1}^{t}\left\{f\left(v_{i}\right)-f\left(v_{i-1}\right)\right\}=n-1=|E(T)| .
$$

Consequently, by (2.1),

$$
P(T) \geq|E(T)|+\sum_{i=1}^{r} \sum_{j=1}^{n_{i}} P\left(T_{i j}\right)=P_{f_{z u}}(T) \geq P(T) .
$$

Therefore, all inequalities in (2.2) to (2.4) are equalities. This implies the following:

(1) each $f_{i j}$ is an optimal labeling for $T_{i j}$,

(2) $w_{f}\left(v_{0}\right)=w_{f}\left(v_{1}\right)=\cdots=w_{f}\left(v_{s}\right)=0$,

(3) $w_{f}\left(v_{t+1}\right)=w_{f}\left(v_{t+2}\right)=\cdots=w_{f}\left(v_{r}\right)=0$,

(4) $f\left(v_{i-1}\right)=\min _{y \in N\left[v_{i}\right]} f(y)$ for $s+1 \leq i \leq t$.

Statement (2) implies that $s=0$, otherwise $w_{f}\left(v_{s-1}\right)=f\left(v_{s-1}\right)-f\left(v_{s}\right)>0$. That is, $x=z$, which is a leaf. Statement (3) implies that $r-1 \leq t$, otherwise either $f\left(v_{t+1}\right)>f\left(v_{t+2}\right)$ or $f\left(v_{t+1}\right)<f\left(v_{t+2}\right)$, i.e., either $w_{f}\left(v_{t+1}\right)>0$ or $w_{f}\left(v_{t+2}\right)>0$. So either $y=u$ or $y=u^{\prime}$. In the former case, $y=u$ is a leaf. In the latter case, $y=u^{\prime}$ is adjacent to at most one non-leaf vertex, otherwise we can choose a longer $\alpha(z, u)$. In this case, since $P_{f_{z u^{\prime}}}(T)=P_{f_{z u}}(T)$, we replace $u$ by $u^{\prime}$ and assume $y=u$. Note that in this case $n_{r}>0$. So, now $\alpha(x, y)=\alpha(z, u)$. Finally, statement (4) implies the following:

(5) $1=f\left(v_{0}\right)<f\left(v_{1}\right)<\cdots<f\left(v_{r}\right)=n$,

(6) $f\left(v_{i-1}\right)<f\left(v_{i j}\right)$ for $1 \leq i \leq r$ and $1 \leq j \leq n_{i}$.

On the other hand, statement (1) and Proposition 2.1 imply that each $f\left(V\left(T_{i j}\right)\right)$ contains consecutive integers. From this, together with statements (5) and (6), we obtain $f=f_{z u}=$ $f_{x y}$. 
COROLlARY 2.5. For any tree $T$ there is an optimal labeling $f_{x y}$ in which both $x$ and $y$ are leaves.

From now on, all optimal labelings we consider are as specified in Corollary 2.5. The path $\alpha(x, y)$ is called a basic path for $P(T)$.

Theorem 2.4 and (2.1) tell us that in order to find the profile of a tree $T$ we need only find a basic path $\alpha(x, y)$ whose deletion results in a forest with the smallest possible profile.

For technical reasons, we now consider the following restricted path deletion problem. Suppose $y$ is a fixed vertex in tree $T$; find a path $\alpha(x, y)$ ending at $y$ such that $P(T-\alpha(x, y))$ is minimum. We use $P^{\prime}(T, y)$ to denote this minimum value. We also call $\alpha(x, y)$ the basic path for $P^{\prime}(T, y)$.

Suppose $f_{x y}$ is an optimal labeling of $T$ and the tree $T$ is as shown in Fig. 2.1. Denote by ${ }^{k} T$ (respectively $T^{k}$ ) the subtree of $T$ that contains $v_{0}, v_{1}, \ldots, v_{k}, F_{1}, \ldots, F_{k}$ (respectively $\left.v_{k}, \ldots, v_{r}, F_{k}, \ldots, F_{r}\right)$. From Theorem 2.4 and (2.1), we obtain the following corollary.

COROLLARY 2.6. For a basic path $\left(v_{0}, v_{1}, \ldots, v_{r}\right)$ for $P(T)$, the following hold:

(1) $\left(v_{0}, v_{1}, \ldots, v_{k}\right)$ is a basic path for $P^{\prime}\left({ }^{k} T, v_{k}\right)$ and $P^{\prime}\left({ }^{k} T, v_{k}\right)=\sum_{i=1}^{k} P\left(F_{i}\right)=$ $\sum_{i=1}^{k} \sum_{j=1}^{n_{i}} P\left(T_{i j}\right)$ for $1 \leq k \leq r$.

(2) $\left(v_{k}, v_{k+1}, \ldots, v_{r}\right)$ is a basic path for $P^{\prime}\left(T^{k}, v_{k}\right)$ and $P^{\prime}\left(T^{k}, v_{k}\right)=\sum_{i=k}^{r} P\left(F_{i}\right)=$ $\sum_{i=k}^{r} \sum_{j=1}^{n_{i}} P\left(T_{i j}\right)$ for $1 \leq k \leq r$.

(3) $P(T)=|E(T)|+P^{\prime}\left({ }^{s} T, v_{s}\right)+P^{\prime}\left(T^{t}, v_{t}\right)+\sum_{i=s+1}^{t-1} P\left(F_{i}\right)$ for $1 \leq s<t \leq r$.

PROPOSITION 2.7. $P(T) \leq P^{\prime}(T, y)+|E(T)|$ for any vertex $y$ in $T$.

3. Main theorems. This section develops theorems that restrict the possibilities of the basic paths for $P(T)$ and $P^{\prime}(T, y)$. In particular, the basic path $\alpha(x, y)$ for $P(T)$ contains the centroids of $T$. We also prove that the basic path for $P^{\prime}(T, u)$ is either $\alpha(x, u)$ or $\alpha(y, u)$, and the deletion of the basic path for $P^{\prime}(T, u)$ from $T$ results in a forest each of whose components has at most $2|V(T)| / 3$ vertices. These results are the keystone of our algorithm for the profile maximization problem.

A centroid of a tree of $n$ vertices is a vertex whose deletion results in a forest each of whose components has at most $\left\lfloor\frac{n}{2}\right\rfloor$ vertices. It is well known that a tree has either exactly one centroid or exactly two adjacent centroids (see [2]). A "from leaves to center" method can be employed to derive the centroids of a tree. This method requires linear time.

THEOREM 3.1. Any basic path $\alpha(x, y)$ for $P(T)$ contains all centroids of $T$.

Proof. Suppose there is a centroid of $T$ not in the basic path

$$
\alpha(x, y)=\left(x, \ldots, v_{1}, u, v_{2}, \ldots, y\right) .
$$

Then $T$ is of the form shown in Fig. 3.1, with $\left|V\left(T^{\prime}\right)\right| \geq n / 2$ where $n=|V(T)|$. By Corollary $2.6(3)$, we have

$$
P(T)=|E(T)|+P^{\prime}\left(T_{1}, v_{1}\right)+P^{\prime}\left(T_{2}, v_{2}\right)+\sum_{i=3}^{k} P\left(T_{i}\right)+P\left(T^{\prime}\right) .
$$

Up to a symmetric argument, we may assume that $\left|V\left(T_{1}\right)\right| \leq\left|V\left(T_{2}\right)\right|$. Let $\alpha(z, v)$ be a basic path for $P\left(T^{\prime}\right)$. Corollary 2.6 (3) and Proposition 2.2 give

$$
P\left(T^{\prime}\right) \geq\left|E\left(T^{\prime}\right)\right|+P^{\prime}\left(T_{a}, a\right)+P^{\prime}\left(T_{b}, b\right)+\sum_{j=1}^{m} P\left(F_{j}\right) .
$$

We also assume that $\left|V\left(T_{a}\right)\right| \leq\left|V\left(T_{b}\right)\right|$. Now consider the labeling $f_{v y}$ for $T$. By (2.1) and Corollary 2.6, we have 
(3.3)

$$
P_{f_{v y}}(T)=|E(T)|+P^{\prime}\left(T_{b}, b\right)+P^{\prime}\left(T_{2}, v_{2}\right)+P\left(T_{a}\right)+\sum_{j=1}^{m} P\left(F_{j}\right)+P\left(T_{1}\right)+\sum_{i=3}^{k} P\left(T_{i}\right) .
$$

Equations (3.1) to (3.3) together lead to that $\left|E\left(T^{\prime}\right)\right| \leq P\left(T_{a}\right)-P^{\prime}\left(T_{a}, a\right)+P\left(T_{1}\right)-P^{\prime}\left(T_{1}, v_{1}\right)$. Then $\left|E\left(T^{\prime}\right)\right| \leq\left|E\left(T_{a}\right)\right|+\left|E\left(T_{1}\right)\right|$ by Proposition 2.7. Thus

$$
\begin{aligned}
\left|E\left(T_{1}\right)\right| & \geq\left|E\left(T^{\prime}\right)\right|-\left|E\left(T_{a}\right)\right| \\
& >\left|E\left(T^{\prime}\right)\right| / 2 \quad\left(\text { since }\left|E\left(T_{a}\right)\right| \leq\left|E\left(T_{b}\right)\right| \text { and } T^{\prime}-\left(T_{a} \cup T_{b}\right) \neq \emptyset\right) \\
& \geq\left(n-\left|E\left(T^{\prime}\right)\right|\right) / 2 \quad\left(\text { since }\left|E\left(T^{\prime}\right)\right| \geq n / 2\right) \\
& \geq\left|E\left(T_{1}\right)\right| \quad\left(\text { since }\left|E\left(T_{1}\right)\right| \leq\left|E\left(T_{2}\right)\right|\right),
\end{aligned}
$$

which is a contradiction.

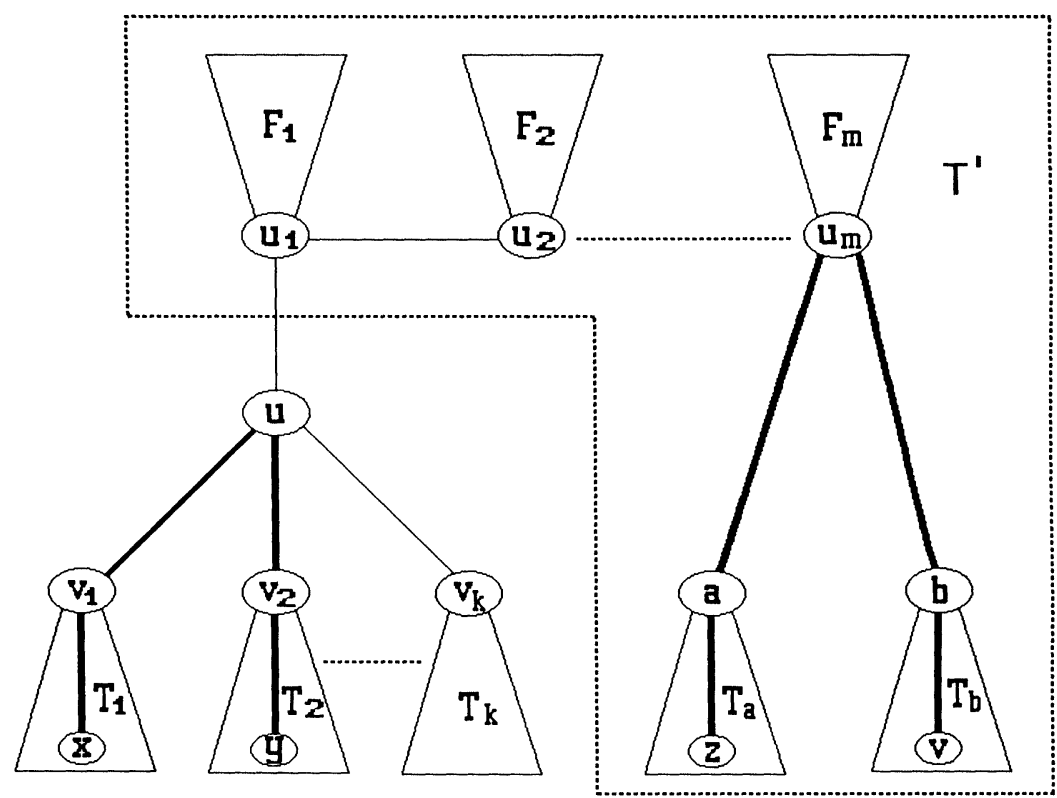

FIG. 3.1 .

Similar arguments lead to the following theorem.

THEOREM 3.2. Suppose $y$ is a fixed vertex of a tree $T$ of $n$ vertices. For any basic path $\alpha(x y)$ of $P^{\prime}(T, y)$, every component of $T-\alpha(x, y)$ has at most $2 n / 3$ vertices.

Proof. The proof of this theorem is exactly the same as that for Theorem 3.1, except now we assume $\left|E\left(T^{\prime}\right)\right|>2 n / 3$ and $\left|E\left(T_{a}\right)\right| \leq\left|E\left(T_{b}\right)\right|$, and there is no assumption that $\left|E\left(T_{1}\right)\right| \leq\left|E\left(T_{2}\right)\right|$. However, we still have $\left|E\left(T^{\prime}\right)\right| \leq\left|E\left(T_{a}\right)\right|+\left|E\left(T_{1}\right)\right|$. Then

$$
\begin{array}{rlr}
\left|E\left(T_{1}\right)\right| & \geq\left|E\left(T^{\prime}\right)\right|-\left|E\left(T_{a}\right)\right| \\
& \geq\left|E\left(T^{\prime}\right)\right| / 2 \quad\left(\text { since }\left|E\left(T_{a}\right)\right| \leq\left|E\left(T_{b}\right)\right|\right) \\
& >n-\left|E\left(T^{\prime}\right)\right| \quad\left(\text { since }\left|E\left(T^{\prime}\right)\right|>2 n / 3\right) \\
& \geq\left|E\left(T_{1}\right)\right|,
\end{array}
$$

which is a contradiction. 
Figure 3.2 gives an example in which a basic path $\alpha(x, y)$ for $P^{\prime}(T, y)$ does not contain the centroid $z$ of $T$.

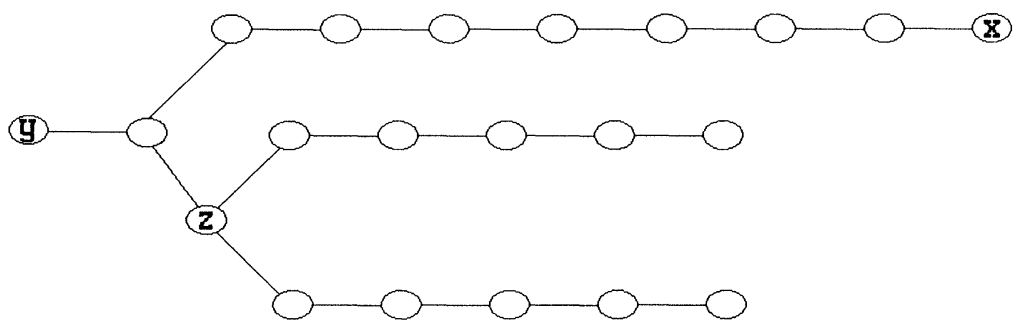

FIG. 3.2.

THEOREM 3.3. If $\alpha(x, y)$ is a basic path for $P(T)$ and $u$ is a fixed vertex in $T$, then either $\alpha(x, u)$ or $\alpha(y, u)$ is a basic path for $P^{\prime}(T, u)$.

Proof. Suppose $\alpha(x, y)=\left(x, \ldots, v_{1}, u_{1}, v_{2}, \ldots, y\right)$ and $\left(u_{1}, u_{2}, \ldots, u_{r}=u\right)$ is the unique path from $\alpha(x, y)$ to $u$, as shown in Fig. 3.3. Let $\alpha(z, u)$ be a basic path for $P^{\prime}(T, u)$.

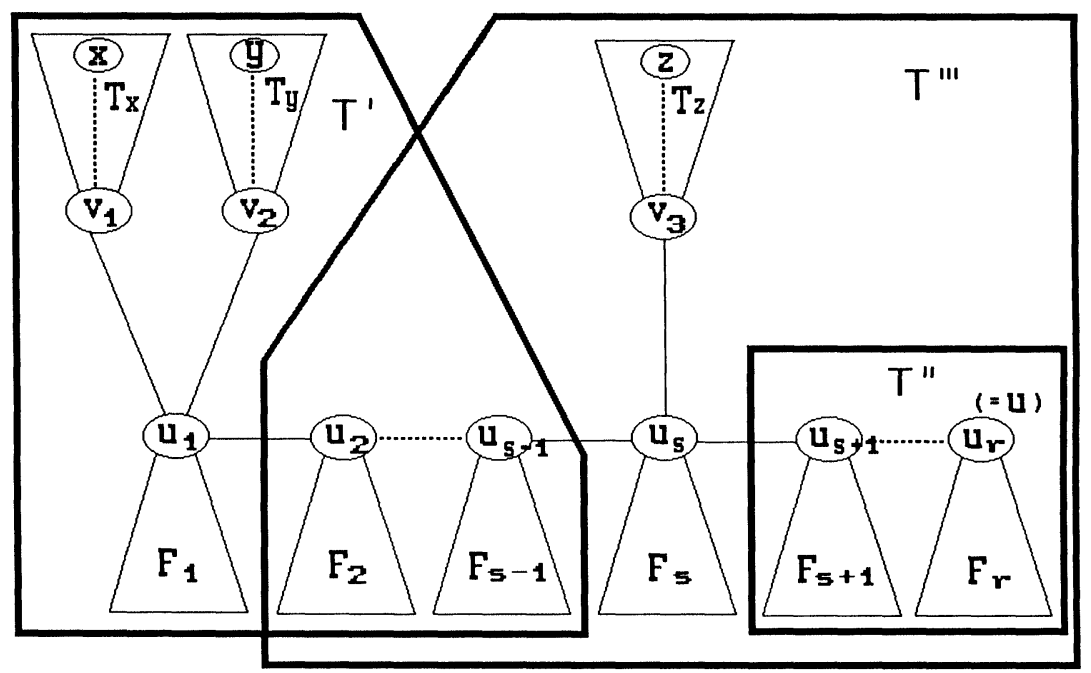

FIG. 3.3.

Case 1. $z \in V\left(T_{x}\right)$. In this case, $u_{s}=u_{1}, v_{3}=v_{1}$, and $T_{z}=T_{x}$. By Corollary $2.6(1)$, $\alpha\left(x, v_{1}\right)$ is a basic path for $P^{\prime}\left(T_{x}, v_{1}\right)$, and so $P\left(T_{x}-\alpha\left(x, v_{1}\right)\right) \leq P\left(T_{z}-\alpha\left(z, v_{3}\right)\right)$. Then

$$
\begin{aligned}
P^{\prime}(T, u) & =P(T-\alpha(z, u)) \\
& =P\left(T_{z}-\alpha\left(z, v_{3}\right)\right)+P\left(T_{y}\right)+\sum_{i=1}^{r} P\left(F_{i}\right) \\
& \geq P\left(T_{x}-\alpha\left(x, v_{1}\right)\right)+P\left(T_{y}\right)+\sum_{i=1}^{r} P\left(F_{i}\right) \\
& =P(T-\alpha(x, u)) .
\end{aligned}
$$

Hence $\alpha(x, u)$ is also a basic path for $P^{\prime}(T, u)$.

Case 2. $z \in V\left(T_{y}\right)$. By a similar argument, $\alpha(y, u)$ is also a basic path for $P^{\prime}(T, u)$. 
Case 3. $z \notin V\left(T_{x}\right)$ and $z \notin T\left(T_{y}\right)$. Let $T^{\prime}, T^{\prime \prime}$, and $T^{\prime \prime \prime}$ be subtrees, as shown in Fig. 3.3. Note that in the case of $s=1, T^{\prime}=T_{x} \cup T_{y}$ is not a tree. Now

$$
P(T-\alpha(z, u))=P^{\prime}\left(T_{z}, v_{3}\right)+P\left(T^{\prime}\right)+\sum_{i=s}^{r} P\left(F_{i}\right) .
$$

Note that $P^{\prime}\left(T_{z}, v_{3}\right)=P\left(T_{z}-\alpha\left(z, v_{3}\right)\right)$. By Proposition 2.2, we have

$$
P\left(T^{\prime}\right) \geq P\left(T_{x}\right)+P\left(T_{y}\right)+\sum_{i=1}^{s-1} P\left(F_{i}\right) .
$$

Since $\alpha(x, y)$ is a basic path for $P(T)$, we have $P_{f_{x z}}(T) \geq P_{f_{x y}}(T)$. By (2.1) and Corollary 2.6 (3) we have

$$
\begin{aligned}
& |E(T)|+P\left(T_{x}-\alpha\left(x, v_{1}\right)\right)+P\left(T_{y}\right)+\sum_{i=1}^{s} P\left(F_{i}\right)+P\left(T^{\prime \prime}\right)+P\left(T_{z}-\alpha\left(z, v_{3}\right)\right) \\
& \geq|E(T)|+P^{\prime}\left(T_{x}, v_{1}\right)+P^{\prime}\left(T_{y}, v_{2}\right)+P\left(F_{1}\right)+P\left(T^{\prime \prime \prime}\right) .
\end{aligned}
$$

Note that $P^{\prime}\left(T_{x}, v_{1}\right)=P\left(T_{x}-\alpha\left(x, v_{1}\right)\right)$. Again, by Proposition 2.2,

$$
P\left(T^{\prime \prime \prime}\right) \geq \sum_{i=2}^{s} P\left(F_{i}\right)+P\left(T_{z}\right)+P\left(T^{\prime \prime}\right) .
$$

Equations (3.4) to (3.7) together lead to

$$
P(T-\alpha(z, u)) \geq P^{\prime}\left(T_{y}, v_{2}\right)+P\left(T_{x}\right)+\sum_{i=1}^{r} P\left(F_{i}\right)+P\left(T_{z}\right)=P(T-\alpha(y, u)) .
$$

Hence $\alpha(y, u)$ is a basic path for $P^{\prime}(T, u)$.

4. The algorithm. We can use the theorems in $\S 3$ to design an efficient algorithm for the profile minimization problem in a tree $T$. By Theorem 3.1, the basic idea of our algorithm is to find a centroid $z$ first in linear time. Suppose $T-z=\cup_{1 \leq i \leq m} T_{i}$, where $u_{i}$ is the only vertex of $T_{i}$ that is adjacent to $z$ in $T$ (see Fig. 4.1). To use Corollary 2.6 (3), we need to find all

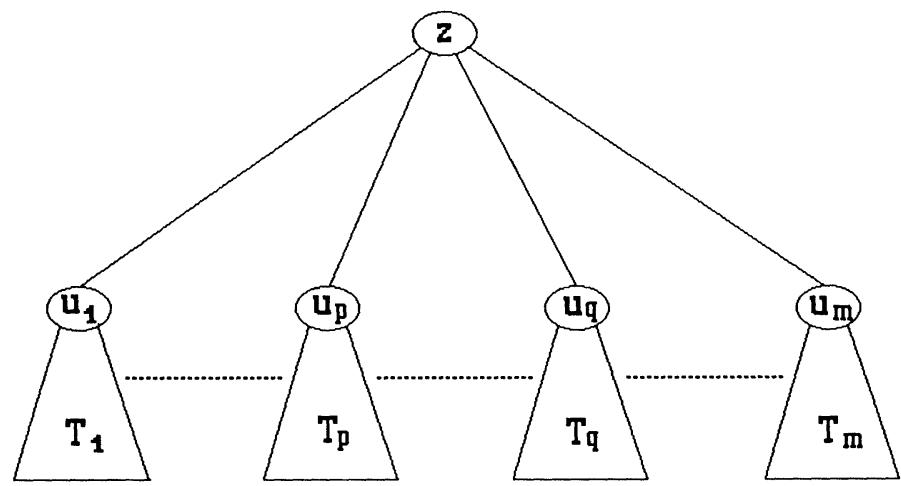

FIG. 4.1. 
profiles $P\left(T_{i}\right)$ and $P^{\prime}\left(T_{i}, u_{i}\right)$ recursively. In the following, Algorithm PROFILE finds $P(T)$ and Algorithm PROFILE1 finds $P^{\prime}(T, u)$. Note that, in order to make use of Theorem 3.3, Algorithm PROFILE not only has to output the value $P(T)$ but also a basic path.

ALGORITHM PROFILE

Input: A tree $T$ of $n$ vertices.

Output: A basic path $\alpha(x, y)=\left(v_{0}, v_{1}, \ldots, v_{r}\right)$ for $P(T)$ and the values $P(T)$ and $P\left(T_{i j}\right)$ for $1 \leq i \leq r-1$ and $1 \leq j \leq n_{i}$.

Method:

1. find a centroid $z$ of $T$.

2. let $T-z=\cup_{1 \leq k \leq m} T_{k}$ and $z$ be adjacent to $u_{k} \in V\left(T_{k}\right)$ for $1 \leq k \leq m$.

3. for each $1 \leq k \leq m$, recursively call PROFILE for $T_{k}$ to get a basic path $\alpha\left(x_{k}, y_{k}\right)$ and values $P\left(T_{k}\right)$ and $P\left(T_{k i j}\right)$, where $T_{k i j}$ are the components of $T_{k}-\alpha\left(x_{k}, y_{k}\right)$.

4. for each $1 \leq k \leq m$, recursively call PROFILE1 for $\left(T_{k}, u_{k}\right)$ to get a basic path $\alpha\left(z_{k}, u_{k}\right)$ and values $P^{\prime}\left(T_{k}, u_{k}\right)$ and $P\left(T_{k i j}^{\prime}\right)$, where $T_{k i j}^{\prime}$ are the components of $T_{k}-$ $\alpha\left(z_{k}, u_{k}\right)$.

5. let $P(T)=n+\min _{1 \leq p<q \leq m}\left\{P^{\prime}\left(T_{p}, u_{p}\right)+P^{\prime}\left(T_{q}, u_{q}\right)+\sum_{i \neq p, q}\left(T_{i}\right)\right\}$, where $p^{*}$ and $q^{*}$ attain the above minimum.

6. let $\alpha(x, y)=\alpha\left(z_{p^{*}}, u_{p^{*}}\right)+z+\alpha\left(u_{q^{*}}, z_{q^{*}}\right)$.

7. combine profiles $P\left(T_{p^{*} i j}\right), P\left(T_{k}\right)$ for $k \neq p^{*}, q^{*}$, and $P\left(T_{q^{*} i j}\right)$ to get profiles $P\left(T_{i j}\right)$.

To find $P^{\prime}(T, u)$, we note that by Theorem 3.3, either $\alpha(x, u)$ or $\alpha(y, u)$ is a basic path for $P^{\prime}(T, u)$. So we consider the configuration in Fig. 3.3 with $T_{z}$ omitted.

\section{ALGORITHM PROFILE1}

Input: Tree $T$ of $n$ vertices with a basic path $\alpha(x, y)=\left(v_{0}, v_{1}, \ldots, v_{r}\right)$ for $P(T)$ and the values $P\left(T_{i j}\right)$ for $1 \leq i \leq r-1$ and $1 \leq j \leq n_{i}$. $u$ is a fixed vertex in $T$.

Output: A basic path $\alpha(z, u)=\left(v_{0}^{\prime}, v_{1}^{\prime}, \ldots, v_{r^{\prime}}^{\prime}\right)$ for $P^{\prime}(T, u)$ and the values $P^{\prime}(T, u)$ and $P\left(T_{i j}^{\prime}\right)$ for $1 \leq i \leq r^{\prime}$ and $1 \leq j \leq n_{i}^{\prime}$.

Method:

1. identify the path $\left(u_{1}, u_{2}, \ldots, u_{r}\right)$ as in Fig. 3.3.

2. recursively use PROFILE to solve $P\left(T_{x}\right), P\left(T_{y}\right), P\left(F_{i}\right)$ (in fact $P\left(T_{i j}\right)$ for each component in $F_{i}$ ) for $1 \leq i \leq r$.

3. $a=P^{\prime}\left(T_{x}, v_{1}\right)+P\left(T_{y}\right)+\sum_{i=1}^{r} P\left(F_{i}\right)$,

$b=P^{\prime}\left(T_{y}, v_{2}\right)+P\left(T_{x}\right)+\sum_{i=1}^{r} P\left(F_{i}\right)$,

where $P^{\prime}\left(T_{x}, v_{1}\right)$ and $P^{\prime}\left(T_{y}, v_{2}\right)$ can be computed from the input values $P\left(T_{i j}\right)$.

4. $P^{\prime}(T, u)=\min \{a, b\}$.

if $a \leq b$ then $z=x$ else $z=y$.

5. combine part of the profiles $P\left(T_{i j}\right), P\left(T_{x}\right)$, or $P\left(T_{y}\right)$, and $P\left(F_{i}\right)$ to get profiles $P\left(T_{i j}^{\prime}\right)$.

5. Time complexity. This section shows that the time complexities of the above two algorithms are $O\left(n^{1.722}\right)$. Let $f(n)$ (respectively, $g(n)$ ) be the time complexity for Algorithm PROFILE (respectively, PROFILE1).

In Algorithm PROFILE, Step 3 (respectively, 4) needs $\sum_{i=1}^{m} f\left(n_{i}\right)$ (respectively, $\sum_{i=1}^{m}$ $\left.g\left(n_{i}\right)\right)$ time, where $n_{i}=\left|V\left(T_{i}\right)\right|$ for $1 \leq i \leq m$. All other steps need $O(n)$ time. Note that for Step 5 we only have to find the smallest and the second smallest values of $P^{\prime}\left(T_{i}, w_{i}\right)-P\left(T_{i}\right)$. Therefore

$$
f(n)=\sum_{i=1}^{m}\left\{f\left(n_{i}\right)+g\left(n_{i}\right)\right\}+c_{1} n,
$$

where 


$$
\sum_{i=1}^{m} n_{i}=n-1 \quad \text { and } \quad n_{i} \leq n / 2 \quad \text { for } \quad 1 \leq i \leq m .
$$

Similarly, in Algorithm PROFILE1, Step 2 needs $\sum_{i=1}^{m} f\left(n_{i}\right)$ time and all other steps need $O(n)$ time. Thus, by Theorem 3.2,

$$
g(n)=\sum_{i=1}^{m} f\left(n_{i}\right)+c_{2} n,
$$

where

$$
\sum_{i=1}^{m} n_{i} \leq n-1 \quad \text { and } \quad n_{i} \leq 2 n / 3 \quad \text { for } \quad 1 \leq i \leq m .
$$

To solve (5.1) and (5.2), we first choose a number $\sigma>1$, which is very close to 1 , say, $\sigma=1.001$. Then choose $1<\lambda<2$ such that

$$
\epsilon=\left(\frac{2}{3}\right)^{\lambda}+\left(\frac{1}{3}\right)^{\lambda}<1
$$

and

$$
\delta=(1+\sigma \epsilon) 2\left(\frac{1}{2}\right)^{\lambda}<1 .
$$

Note that a simple computer program gives that $\lambda=1.722$ for $\sigma=1.001$.

THEOREM 5.1. There exists a constant $c$ such that $f(n) \leq c n^{\lambda}$ and $g(n) \leq c \sigma \epsilon n^{\lambda}$ for all $n$, i.e., $f(n)=O\left(n^{\lambda}\right)$ and $g(n)=O\left(n^{\lambda}\right)$.

Proof. The proof is by induction on $n$. Assume that there exists a constant $c$ such that $f\left(n^{\prime}\right) \leq c n^{\prime \lambda}$ and $g\left(n^{\prime}\right) \leq c \sigma \epsilon n^{\prime \lambda}$ for all $n^{\prime}<n$. We also assume that $c \geq c_{1} /(1-\delta)$ and $c \geq c_{2} /(\sigma-1) \epsilon$.

For $0<a \leq b$, consider the function $h(x)=(b+x)^{\lambda}+(a-x)^{\lambda}-b^{\lambda}-a^{\lambda}$ where $0 \leq x \leq a$. Note that $h^{\prime}(x)=\lambda(b+x)^{\lambda-1}-\lambda(a-x)^{\lambda-1} \geq 0$. So $h$ is an increasing function and then $h(x) \geq h(0)=0$ for $0 \leq x \leq a$. Thus

$$
b^{\lambda}+a^{\lambda} \leq(b+x)^{\lambda}+(a-x)^{\lambda} \text { for } 0 \leq x \leq a \leq b .
$$

By (5.1) and the induction hypothesis, we have $f(n) \leq c(1+\sigma \epsilon) \sum_{i=1}^{m} n_{i}^{\lambda}+c_{1} n$ where $\sum_{i=1}^{m} n_{i}=n-1$ and $n_{i} \leq n / 2$ for $1 \leq i \leq m$. Repeatedly apply (5.3) to get $\sum_{i=1}^{m} n_{i}^{\lambda} \leq$ $\left(\frac{n}{2}\right)^{\lambda}+\left(\frac{n}{2}-1\right)^{\lambda}$. Therefore $f(n) \leq c(1+\sigma \epsilon) 2\left(\frac{1}{2}\right)^{\lambda} n^{\lambda}+c_{1} n=c \delta n^{\lambda}+c_{1} n$. By the choice of $c, c_{1} n \leq c(1-\delta) n \leq c(1-\delta) n^{\lambda}$. Then $f(n) \leq c n^{\lambda}$.

By (5.2) and the induction hypothesis, we have $g(n) \leq c \sum_{i=1}^{m} n_{i}^{\lambda} c_{2} n$ where $\sum_{i=1}^{m} n_{i} \leq$ $n-1$ and $n_{i} \leq 2 n / 3$ for $1 \leq i \leq m$. Repeatedly apply (5.3) to get $\sum_{i=1}^{m} n_{i}^{\lambda} \leq(2 n / 3)^{\lambda}+$ $\left(\frac{n}{3}\right)^{\lambda}=\epsilon n^{\lambda}$. Also, by the choice of $c, c_{2} n \leq c(\sigma-1) \epsilon n \leq c(\sigma-1) \epsilon n^{\lambda}$. Then $g(n) \leq$ $c \epsilon n^{\lambda}+c(\sigma-1) \epsilon n^{\lambda}=c \sigma \epsilon n^{\lambda}$.

Acknowledgments. We thank the two anonymous referees for many useful suggestions on the revision of this paper. 


\section{REFERENCES}

[1] D. Adolphson and T. C. Hu, Optimal linear ordering, SIAM J. Appl. Math., 25 (1973), pp. $403-423$.

[2] F. BuCKLEY AND F. HARARY, Distance in Graphs, Addison-Wesley, Reading, MA, 1990.

[3] F. R. K. Chung, On optimal linear arrangements of trees, Comput. Math. Appl., 10 (1984), pp. $43-60$.

[4] M. R. GAREY AND D. S. Johnson, Computer and Intractability: A Guide to the Theory of NP-Completeness, Freeman, San Francisco, CA, 1979.

[5] Y.Lin AND J. YUAN, Profile minimization problem for matrices and graphs, preprint, Dept. of Math., Zhengzhou University, People's Republic of China, 1990.

[6] - Minimum profile of grid networks in structure analysis, to appear.

[7] Y. SHILOACH, A minimum linear arrangement algorithm for undirected tree, SIAM J. Comp., 8 (1979), pp. 15-32.

[8] R. P. Tewarson, Sparse Matrices, Academic Press, New York, 1973.

[9] O. C. ZIENKIEWICZ, Finite Element Method in Engineering Science, McGraw Hill, London, 1971. 